\title{
Diagnostics du panache d'ablation
}

\author{
M. Autric
}

\author{
IRPHE, Institut de Recherche sur les Phénomènes Hors Équilibre, UMR 6594 du CNRS, \\ Universités d'Aix-Marseille l et II, Laboratoire LP3 Laser, Plasma et Procédés Photoniques, \\ Parc Scientifique et Technologique de Luminy, Case 918, avenue de Luminy, 13009 Marseille, \\ France
}

\begin{abstract}
Résumé: Les processus hydrodynamiques existant lors des phases d'irradiation de matériaux solides régissent en fait le couplage rayonnement - matière et donc, les effets résultants sur la surface. Déterminer quels processus interviennent dans telles ou telles applications permet de comprendre comment l'énergie laser est couplée au matériau et, éventuellement, d'ajuster les conditions expérimentales afin d'optimiser les procédés. Caractériser les régimes hydrodynamiques, c'est déterminer l'instant de formation du plasma, son évolution spatio-temporelle, la structure des ondes de choc et des ondes d'absorption créées dans le milieu ambiant et leur évolution. Ces différents régimes se caractérisent par des méthodes optiques de visualisation de type ombroscopie, strioscopie et interférométrie.
\end{abstract}

\section{GENERALITES}

L'étude des phénomènes d'interaction rayonnement laser - matière est primordiale pour l'optimisation de bons nombres de procédés industriels. Ces phénomènes sont complexes et liés entre eux tout au long d'une interaction, rendant l'étude de chacun d'eux séparément extrêmement délicate. Les principales étapes à prendre en compte sont : dans un premier temps, l'absorption du rayonnement par la matière, puis, le chauffage d'un élément de volume et la rupture des liaisons du matériau, l'éjection de matière sous forme de vapeur ou de liquide, l'ionisation de la vapeur et la formation d'un plasma lumineux. Celui-ci peut être plus ou moins absorbant, se propager plus ou moins vite en aval de la surface et induire des modifications au niveau des effets escomptés sur le matériau. Tous ces phénomènes vont dépendre des paramètres laser (principalement, longueur d'onde, densité de puissance incidente, durée d'irradiation) et du milieu ambiant (vide ou nature et pression du gaz environnant).

Il est donc primordial de diagnostiquer un panache d'ablation : - * en termes de caractéristiques physiques telles que la densité, la température des espèces composant le plasma, le coefficient d'absorption, le degré d'ionisation, l'énergie interne du plasma, etc...; - * en termes de formes et de structures (expansion axiale et radiale dépendant des régimes hydrodynamiques particuliers).

\section{PRINCIPAUX REGIMES HYDRODYNAMIQUES}

\subsection{Onde de souffle}

Rappel : Lorsqu'on irradie un matériau placé dans l'atmosphère avec une impulsion laser de courte durée, on observe au-dessus du plasma, la formation d'une onde de choc totalement transparente au rayonnement laser. Cette onde de choc appelée onde de souffle résulte d'un dépôt d'énergie instantané dans un volume infinitésimal et d'une compression du milieu ambiant provoquée par l'éjection de particules à partir de la surface irradiée. Le transfert d'énergie du matériau au milieu ambiant est essentiellement de type collisionnel. Ce dépôt d'énergie peut s'effectuer en un point du milieu, le long d'une ligne ou sur une surface donnant naissance à une onde respectivement sphérique, cylindrique ou plane.

A l'aide de l'analyse adimensionnelle, Sedov [1] propose une relation liant l'évolution du rayon de l'onde à l'énergie déposée $\mathrm{E}$, à la densité du gaz au repos et au temps; la vitesse de l'onde est donnée par l'expression suivante : 


$$
\frac{\mathrm{dR}}{\mathrm{dt}}=\frac{2}{3+v} \xi_{0}\left(\frac{\mathrm{E}}{\rho}\right)^{\frac{1}{3+v}} \cdot \mathrm{t}^{-\frac{1+v}{3+v}} \quad \text { avec } \quad \xi_{0}=\left(\frac{75(\gamma-1)(\gamma+1)}{16 \pi(3 \gamma-1)}\right)^{\frac{1}{3+v}} \text { et } \gamma: \text { coefficient d'adiabaticité }
$$

du gaz, $\mathrm{E}$ : énergie déposée, $\rho$ : densité du gaz au repos, $v=0$ (onde plane), $=1$ (onde cylindrique), $=2$ (onde sphérique)

\subsection{Onde d'Absorption entretenue par Laser ( LSA Wave)}

Le plasma peut devenir fortement absorbant : ce plasma très énergétique va alors échanger de l'énergie avec le milieu ambiant suivant des mécanismes de conduction thermique, transfert radiatif ou chauffage par le passage de l'onde de choc. Suivant l'intensité laser, la longueur d'onde et la pression du milieu ambiant, on peut alors observer les principaux régimes d'onde d'absorption [2] : a ) - Onde de combustion entretenue par laser (LSC) [caractérisée par un choc précédant un front d'absorption] cette onde se propage avec une vitesse subsonique dans le gaz "choqué" et une vitesse supersonique par rapport au gaz ambiant ; une expression approchée de la vitesse est $\left(V_{L S C}-U_{2}\right) / U_{2}=0.012 \sqrt{I_{0}}$ pour $I_{0}$ compris entre 1 et $20 \mathrm{MW} / \mathrm{cm}^{2} ; \mathrm{b}$ ) - Onde de détonation entretenue par laser (LSD) [caractérisée par la juxtaposition du choc et du front d'absorption] ; l'augmentation de l'intensité provoque un accroissement de la température derrière le choc (gaz choqué chauffé). Cette température atteint une valeur suffisamment élevée pour que l'absorption du rayonnement laser s'effectue juste derrière le front de choc sans qu'il soit nécessaire qu'il y ait un chauffage additionnel par transfert d'énergie depuis le plasma. La zone d'absorption suit l'onde de choc et se déplace à la même vitesse. Ceci est analogue à une onde de détonation chimique. La vitesse est proportionnelle à $\left.I_{0} / \rho{ }^{1 / 3} \Rightarrow V_{L S D-1 D}=\left[2\left(\gamma_{3}^{2}-1\right) \frac{I_{0}}{\rho_{1}}\right]^{1 / 3} ; c\right)$ - Onde de radiation (LSR) [ existe pour une très forte densité de puissance ; propagation caractérisée par un transfert radiatif très important].

\section{VISUALISATIONS DU PANACHE D'ABLATION}

Différentes méthodes permettent d'identifier les espèces présentes dans un panache d'ablation (atomes, ions, molécules, nanoagrégats) et d'accéder aux grandeurs caractéristiques citées précédemment; il s'agit des méthodes interférométriques (interféromètre de Mach-Zenhder, de Michelson, de Jamin, ...) et des techniques spectroscopiques basées sur l'observation ou l'excitation d'espèces (spectroscopie d'émission, de fluorescence induite par laser, d'absorption, de masse, de temps de vol, ...). Pour ce qui concerne l'analyse de la forme et de la structure interne du panache, il faut avoir recours à des méthodes de visualisations performantes [3,4] : - soit par imagerie CCD intensifiée, monoexposition avec répétition des tirs laser pour couvrir une séquence complète (avantages : temps de pause extrêmement court et très haute sensibilité ; inconvénient : reproductibilité des prises de vues dues aux variations éventuelles des paramètres laser tir à tir); - soit par caméra électronique rapide à convertisseurs d'images (avantages : séquences d'images ou balayage de fente permettant un suivi en continu du phénomène sur un seul tir laser, excellente résolution spatiale ; inconvénient : moins bonne détectivité/sensibilité).

De plus, des montages optiques particuliers du type ombroscopie et strioscopie permettent la visualisation des ondes de choc, des ondes de détente, des ondes d'absorption et de leurs vitesses d'expansion; en effet, ces méthodes consistent à mettre en évidence les fluctuations d'indice de réfraction du milieu, fluctuations liées aux variations de température, de densité et de pression. Les variations de ces grandeurs dans le plan objet (panache d'ablation) seront caractérisées par des modulations d'intensité lumineuse dans le plan image. Il est également possible de caractériser la phase vapeur du panache par absorption résonante; la technique TLRAP "tuning laser resonance absorption photography" consiste à éclairer le panache au moyen d'un laser sonde accordable en fréquence dont la longueur d'onde est ajustée sur une transition spectroscopique de l'élément irradié. On dispose ainsi d'un outil de diagnostic intéressant pour visualiser la dynamique des espèces non - émissives. Des exemples de diagnostics de panache d'ablation sont présentés pour des irradiations de matériaux métalliques et céramiques placés dans différentes conditions d'expériences - sous vide, dans différentes ambiances gazeuses à pression variable - au moyen de laser émettant dans le domaine de l'ultraviolet. 

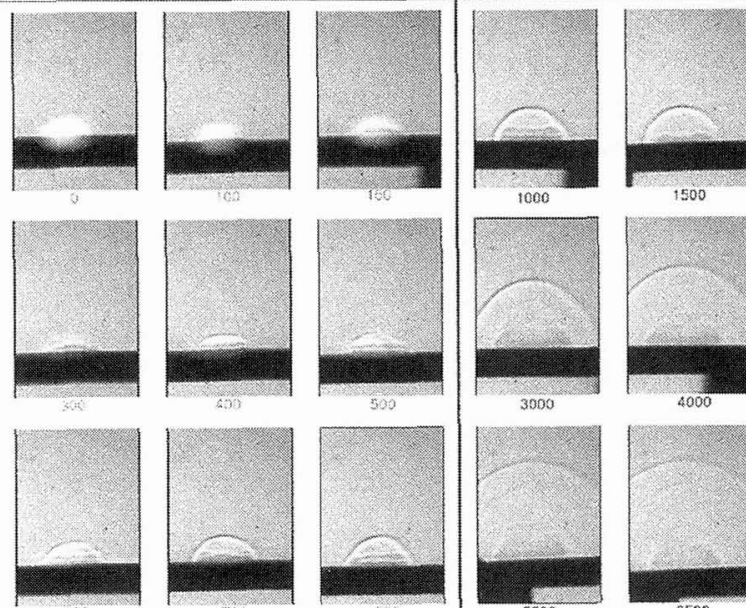

3000
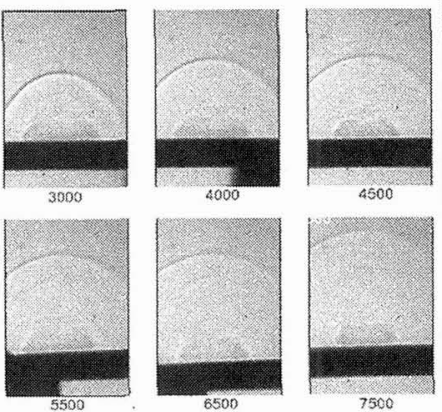
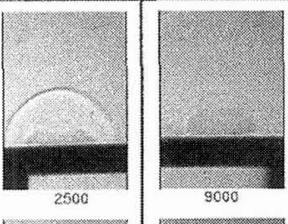

4500
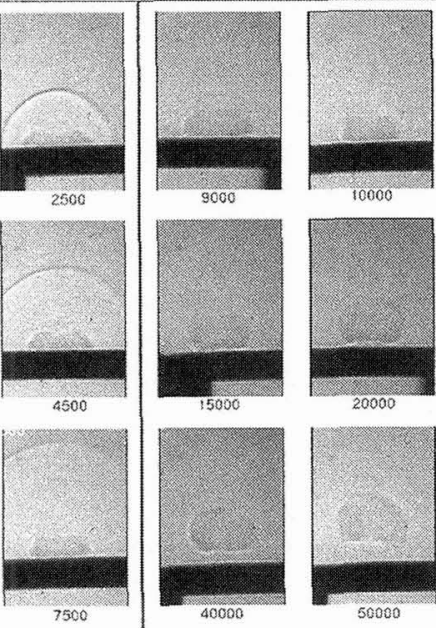
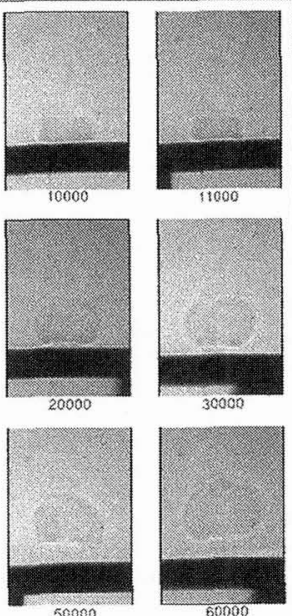

Figure 1 : Onde de choc et plasma sur Carbure de Silicium; fluence $0.8 \mathrm{~J} / \mathrm{cm}^{2}, 20 \mathrm{~ns}$ à $248 \mathrm{~nm}$.

La figure 1 représente l'expansion d'un plasma créé sur un échantillon de $\mathrm{SiC}$; les photographies sont prises à l'instant indiqué sous chaque enregistrement, au moyen d'une caméra $\mathrm{CCD}$ intensifiée avec un temps d'exposition de $5 \mathrm{~ns}$; le montage ombroscopique utilisé permet de mettre en évidence l'onde de choc, de détente, la surface de contact séparant le plasma dense du gaz "choqué, les structures complexes, un panache instable qui se détache de la surface 15 à $20 \mu$ s après la fin de l'irradiation. En combinant un montage interférométrique de type Mach-Zenhder et ombroscopique, il est possible, au moyen d'une caméra électronique rapide, de visualiser plusieurs images sur un même tir laser; les photographies cidessous représentent des séquences d'expansion pour différentes conditions expérimentales. La figure $2 \mathrm{a}$ met en évidence le développement d'un plasma créé sur alliage de titane par une impulsion laser $248 \mathrm{~nm}$ de $300 \mathrm{~ns}$ de durée (fluence $=100 \mathrm{~J} / \mathrm{cm}^{2}$ ) ; la première photo (en haut à gauche) est prise à $150 \mathrm{~ns}$; l'intervalle de temps entre chaque enregistrement est de $50 \mathrm{~ns}$ avec un temps d'exposition de $10 \mathrm{~ns}$. Les prises de vues $2 \mathrm{~b}$ et $2 \mathrm{c}$ ont été obtenues dans l'argon (fluence $=90 \mathrm{~J} / \mathrm{cm}^{2}, \mathrm{t}_{0}=350 \mathrm{~ns}, \Delta \mathrm{t}=50 \mathrm{~ns}$, pression $10^{2}$ $\mathrm{Pa}$ ) et dans l'hélium (fluence $=95 \mathrm{~J} / \mathrm{cm}^{2}, \mathrm{t}_{0}=550 \mathrm{~ns}, \Delta \mathrm{t}=50 \mathrm{~ns}, \mathrm{P}=10^{5} \mathrm{~Pa}$ ). Elles montrent clairement la différence de transparence de ces panaches d'ablation par rapport au milieu gazeux dans lesquels ils ont été créés. Ces prises de vues permettent de déterminer les vitesses, axiale et radiale, des plasmas initiés par laser. La figure 3 montre un bon accord entre les résultats expérimentaux et les modèles d'expansion théoriques des ondes LSD et des ondes de souffle.

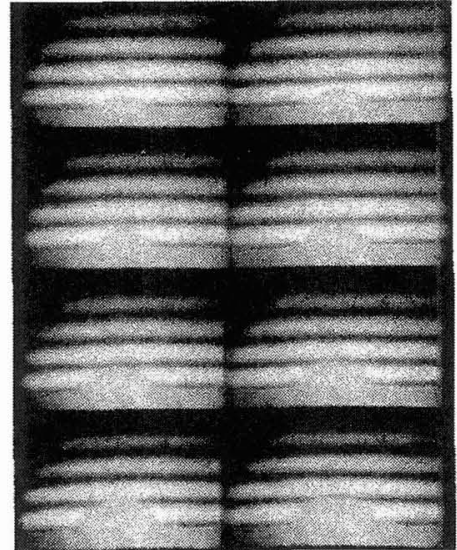

a)

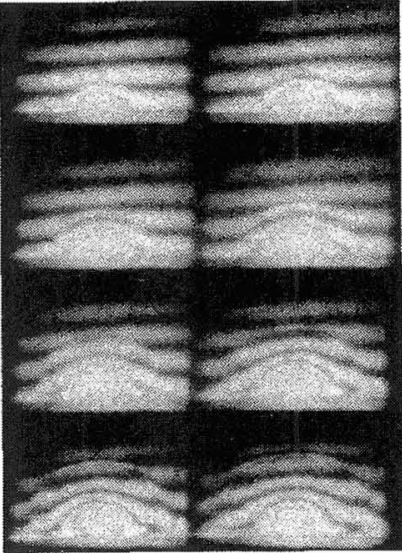

b)

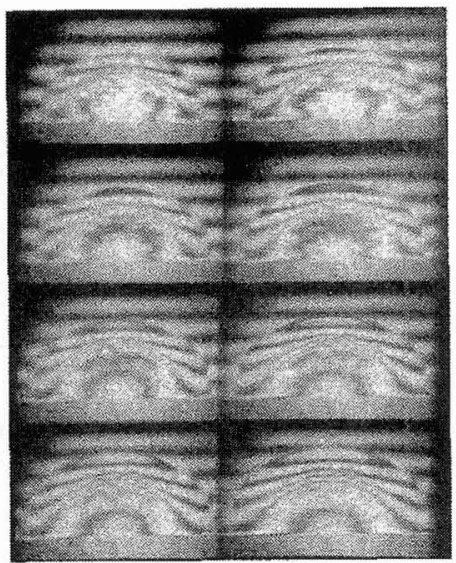

c)

Figure 2 : Visualisations du panache plasma pour différentes conditions expérimentales. 


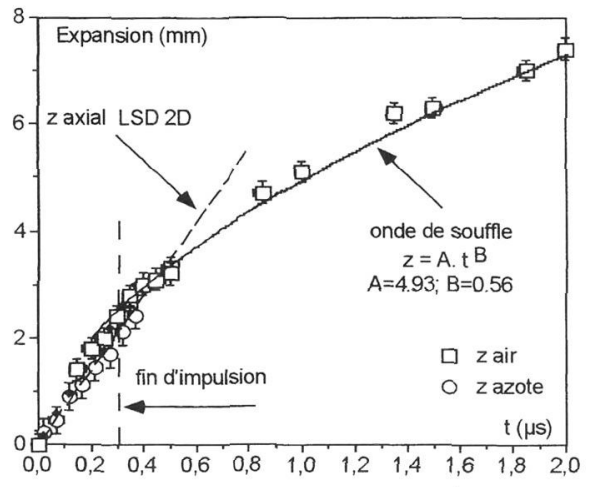

Figure 3 : Expansion axiale du front d'absorption créé sur un échantillon de fitane TA6V dans une atmosphère d'azote et d'air, comparaison avec modèle LSD $2 \mathrm{D}$ et onde de souffle ; $P=10^{5} \mathrm{~Pa}, \mathrm{I}=3.10^{8} \mathrm{~W} / \mathrm{cm}^{2}$.

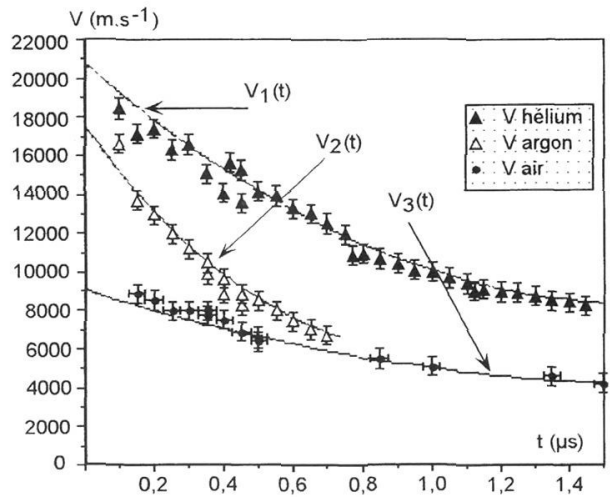

Figure 4 : Vitesse moyenne du front d'absorption créé sur titane TA6V dans une atmosphère d'hélium, d'argon et d'air ; $\lambda=248 \mathrm{~nm}, \mathrm{P}=10^{5} \mathrm{~Pa}, \mathrm{I}=3.10^{8} \mathrm{~W} / \mathrm{cm}^{2}$.

Sur la figure 4 , on remarque que la vitesse axiale du front d'absorption dans l'air est nettement inférieure aux vitesses observées sous hélium ou argon; elle est de l'ordre de $8000 \mathrm{~m} \cdot \mathrm{s}^{-1}$ pendant le régime LSD (250ns) puis ralentit après l'impulsion pour atteindre une valeur de $2700 \mathrm{~m} . \mathrm{s}^{-1}$ au bout de 600 ns. La figure 5 ci-dessous combine la technique de prises de vues par balayage de fente, en interférométrie et ombroscopie. Cela permet de suivre le phénomène, continûment au cours du temps, durant l'interąetion. De plus, en utilisant un laser accordable en fréquence, il est possible de sélectionner une espèce, et une seule, non-émissive, puis de suivre la dynamique de cette espèce sur toute la durée de vie du panache. Dans le cas présent, une transition du titane vapeur à $5145,4 \AA$ est visualisée par absorption résonante d'une raie laser à $5145,3 \AA$.

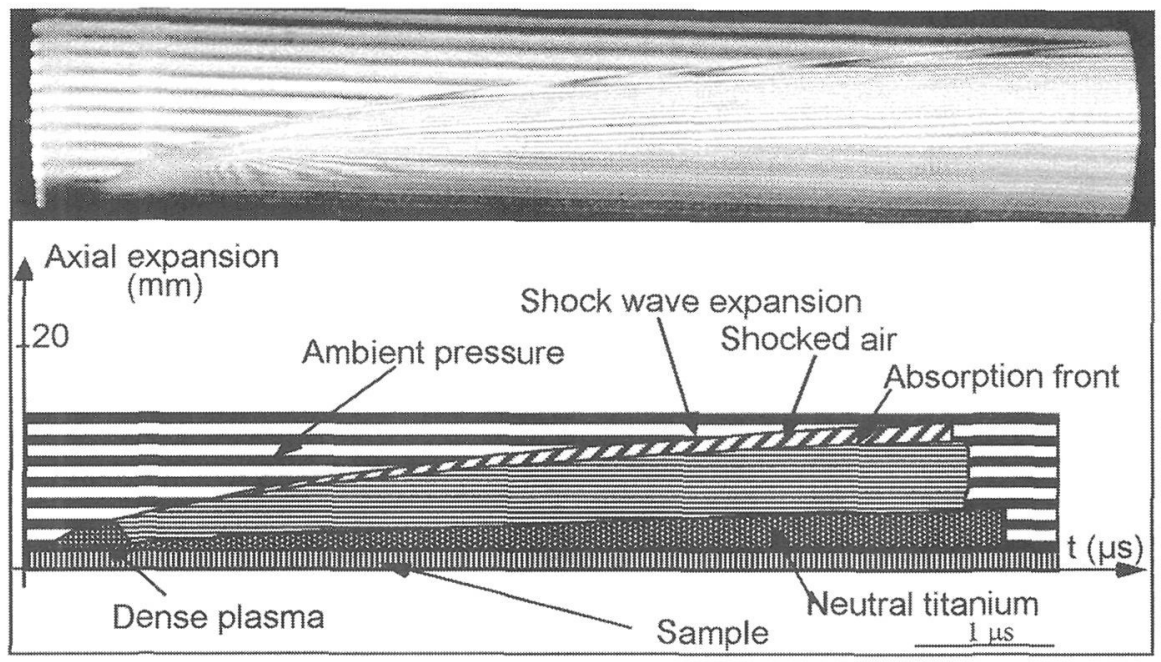

Figure 5 : visualisation en balayage de fente (streak mode) et absorption résonante d'un plasma d'air et de titane fluence $=90 \mathrm{~J} / \mathrm{cm}^{2} ;$ l'axe des abscisses correspond à une durée d'environ $7 \mu \mathrm{s}$.

\section{Références}

1. L.Sedov, Similitude et dimensions en mécanique, éditions Mir, Chap.4, p. 232-277, 1977

2. A. Pirri, R. Root, AlAA Journal, 16 (12), p. 1296-1304, 1978

3. T. Sarnet,. Thèse de Doctorat de l'Université de la Méditerranée (Aix-Marseille II), 1995

4. G. Nicolas-Costa, Thèse de Doctorat de l'Université de Provence (Aix-Marseille I), 1997 